\title{
Myrica esculenta Buch.-Ham. ex D. Don: A Natural Source for Health Promotion and Disease Prevention
}

\author{
Atul Kabra ${ }^{1,2}$, Natália Martins ${ }^{3,4, * \mathbb{C}}$, Rohit Sharma ${ }^{5}$, Ruchika Kabra ${ }^{2}$ and \\ Uttam Singh Baghel ${ }^{2, *(D)}$ \\ 1 I.K. Gujral Punjab Technical University, Kapurthala, Punjab 144603, India; atul.kbr@gmail.com \\ 2 Kota College of Pharmacy, Kota Rajasthan 325003, India; ruchika.p88@gmail.com \\ 3 Faculty of Medicine, University of Porto, Porto 4200-319, Portugal \\ 4 Institute for Research and Innovation in Health (i3S), University of Porto, Porto 4200-135, Portugal \\ 5 Central Ayurveda Research Institute for Drug Development, CCRAS, Ministry of AYUSH, Government of \\ India, Bidhannagar, Kolkata, West Bengal 700091, India; dhanvantari86@gmail.com \\ * Correspondence: ncmartins@med.up.pt (N.M.); uttamsingh1985@gmail.com (U.S.B.)
}

Received: 24 April 2019; Accepted: 25 May 2019; Published: 31 May 2019

\begin{abstract}
Myrica esculenta (Myricaceae) is a popular medicinal plant most commonly found in the sub-tropical Himalayas. It is widely used in folk medicine to treat several ailments such as asthma, cough, chronic bronchitis, ulcers, inflammation, anemia, fever, diarrhea, and ear, nose, and throat disorders. Due to its multidimensional pharmacological and therapeutic effects, it is well recognized in the ayurvedic pharmacopeia. However, the recent upsurge in M. esculenta use and demand has led to illicit harvesting by the horticultural trade and habitat loss, pushing the plant to the brink of extinction. Thus, the present review aims to provide updated information on M. esculenta botany, ethnomedicinal uses, phytochemistry, pharmacological effects, toxicity, and conservation methods, as well as also highlight prospective for future research. Particular emphasis is also given to its antioxidant potential in health promotion. In-depth literature was probed by searching several sources via online databases, texts, websites, and thesis. About 57 compounds were isolated and identified from $M$. esculenta, and the available reports on physicochemical parameters, nutritional and high-performance thin-layer chromatography analysis of bioactive plant parts are portrayed in a comparative manner. Friendly holistic conservation approaches offered by plant biotechnology applications, such as micropropagation, germplasm preservation, synthetic seed production, and hairy root technologies are also discussed. Nonetheless, further studies are needed to propose the mechanistic role of crude extracts and other bioactives, and even to explore the structure-function relationship of active components.
\end{abstract}

Keywords: Myrica esculenta; kaphal; ethnomedicinal; phytoconstituents; conservation; micropropagation; pharmacology

\section{Introduction}

Genus Myrica is a large group comprising more than 97 species in the Myricaceae family. This family contains woody plants native to the subtropical and temperate zones of the earth [1]. Plant species of this genus are distributed in China, Taiwan, Japan, Western Highland of Cameroon, North America, South Africa, Australia, Brazil, Ethiopia, Nepal, and India [2-5]. Specifically, Myrica esculenta Buch.-Ham. ex. D. Don named 'Hairy Bayberry' and widely known as Kaiphal or Kataphala in the Indian subcontinent, is broadly used in Ayurveda (traditional Indian system of medicine) [6-8]. But this plant also has other synonyms, such as Myrica sapida Wall. and Myrica farqhariana Wall. [5,9,10]. Myrica plants grow well in nitrogen-depleted soils, mixed forests, agricultural and marginal lands $[1,11]$. 
Morella esculenta (Buch.-Ham. ex. D. Don) I.M. Turner is the newly accepted name for Myrica esculenta Buch.-Ham. ex.D. Don, and the later name is treated as a basionym of Morella esculenta. Taxonomical classification of Myrica esculenta is Kingdom: Plantae; Phylum: Tracheophyta; Class: Magnoliopsida; Order: Fagales; Family: Myricaceae; Genus: Morella [12].

M. esculenta is known for its edible fruits and other by-products. Indeed, its fruits have been a potential income generating source for the local tribes of the Meghalaya and sub-Himalayan region $[13,14]$. It is likewise known by a variety of names, such as "Katphal" in Sanskrit, "Kaiphal" in Urdu, "Nagatenga" in Assam, 'Soh-phi' in Khasi and 'Box myrtle' in English [1,15-17]. All the parts of the M. esculenta plant have huge nutritional and therapeutic importance. Fruits are used for syrups, jams, pickles, and preparation for refreshing drinks [14]. Traditionally, its bark, roots, and leaves are used for the treatment of various ailments and disorders [3,5]. Besides its traditional uses, bark is also used for making paper and ropes [18]. In addition, $M$. esculenta fruits and roots are used as an active botanical ingredient in numerous ayurvedic formulations (Table 1).

Table 1. Ayurvedic formulations of the plant with their uses and manufacturers.

\begin{tabular}{cccc}
\hline Formulation & Uses & Manufacturers & References \\
\hline “Chwayanprash" & $\begin{array}{c}\text { Improved digestion and strength and } \\
\text { enhanced energy }\end{array}$ & $\begin{array}{c}\text { Dabur, Patanjali, Nature } \\
\text { \& Nurture Healthcare }\end{array}$ & {$[19,20]$} \\
\hline "Katphaladi Churna" & $\begin{array}{c}\text { Treatment of fever, throat infection, } \\
\text { respiratory disorders, and abdominal } \\
\text { pain }\end{array}$ & VHCA Ayurveda & {$[19,20]$} \\
\hline "Pushyanuga Churna" & $\begin{array}{c}\text { Treatment for bleeding disorders and } \\
\text { candidiasis }\end{array}$ & Ayurveda,Baidyanat-h & {$[19,20]$} \\
\hline "Katphala Taila" & Treatment of joint pain & VHCA Ayurveda & {$[19,20]$} \\
\hline "Arimedadi Taila" & $\begin{array}{c}\text { Helps to relieve tooth decay and } \\
\text { breath problem }\end{array}$ & IMIS Pharmaceuticals & {$[19,20]$} \\
\hline "Mahavisagarbha Taila" & $\begin{array}{c}\text { Used for vata imbalance, } \\
\text { neuromuscular conditions }\end{array}$ & VHCA Ayurveda & {$[19,20]$} \\
\hline "Bala Taila" & $\begin{array}{c}\text { Treatment of vata disorders, } \\
\text { respiratory infections and weakness }\end{array}$ & Patanjali & {$[19,20]$} \\
\hline "Khadiradi Gutika" & $\begin{array}{c}\text { Treatment of dental, oral, throat and } \\
\text { tonsillar infections }\end{array}$ & Zandu & {$[19,20]$} \\
\hline "Maha Vatagajankusa & $\begin{array}{c}\text { Rheumatoid arthritis, Migraine, } \\
\text { Paralysis, Cough, Cold, Asthma }\end{array}$ & $\begin{array}{c}\text { Dabur, Baidyanath, } \\
\text { Shree Dhootapapeshwar }\end{array}$ & {$[19,20]$} \\
\hline "Brihat Phala Ghrta" & Treatment of infertility & SN Pandit Ayurvedic & {$[19,20]$} \\
\hline
\end{tabular}

More recently, its numerous ethnomedicinal uses led researchers to explore M. esculenta phytochemistry further. For instance, tannins extracted from its bark are used as a dyeing agent [6]. Indeed, the presence of distinct bioactive compounds, such as alkaloids, flavonoids, glycosides, tannins, terpenoids, saponins, and volatile oils $[8,21]$, has been increasingly reported as related to its pharmacological effects. For example, crude extracts and isolated compounds from M. esculenta exhibit both in vivo and in vitro pharmacological activities. Local tribes use the tree for timber, fuel, fodder, wood, likewise as used for tanning and getting yellow colored dye [22-25]. In spite of being a useful tree, the cultivation of the plant is incredibly restricted, and most of the traditional and commercial uses of M. esculenta rely solely on collections from the wild sources by endemic people [26]. Thus, wild sources of the species are underneath impending danger of extinction due to the increase in urbanization, overharvesting, negligence of sustainable use, and over-exploitation of forests and wastelands for industrial uses [27]. Due to the over-exploitation of the natural habitat, limited geographical prevalence and the unresolved problems inherent in seed vitality and germination, alternative propagation and conservation approaches are desperately needed to avoid the possible extinction of this vital species [8]. This species is fundamentally the same as M. rubra, which is ordinarily found in China and 
Japan. However, M. esculenta contains fruits smaller than about $4-5 \mathrm{~mm}$ compared to the $M$. rubra fruits $(12-15 \mathrm{~mm})$ [28]. Although information on phenolic content and antioxidant activity of the fruit extract, juice, jam and marc of M. rubra [19,20,29-32] is available, this information is lacking for M. esculenta. Previous reviews have suggested that myricetin is obtained mainly by members of the Myricaceae family [33,34] and is a key ingredient in many foods, besides to be used as a food additive due to its antioxidant activity and ability to protect lipids from oxidative damage [35]. It is one of the key ingredients of various foods and beverages. The compound has a wide range of potentialities that include strong antioxidant, anticancer, antidiabetic and anti-inflammatory effects, and can protect a wide variety of cells from in-vitro and in vivo lesions [36]. It was first isolated in the late eighteenth century from the bark of Myrica nagi Thunb. (Myricaceae), harvested in India, as light-yellow crystals [37].

In this sense, this review investigates the relevant information on botanical description, ethnomedicinal uses, phytochemistry, antioxidant activity, pharmacological activity and toxicity, along with conservation of $M$. esculenta. Its critical aspects as a natural source of antioxidant compounds for health promotion and disease prevention are also raised.

\section{Research Methodology}

The research methodology adopted for the selection of articles for this review is stipulated as flow chart in Figure 1.

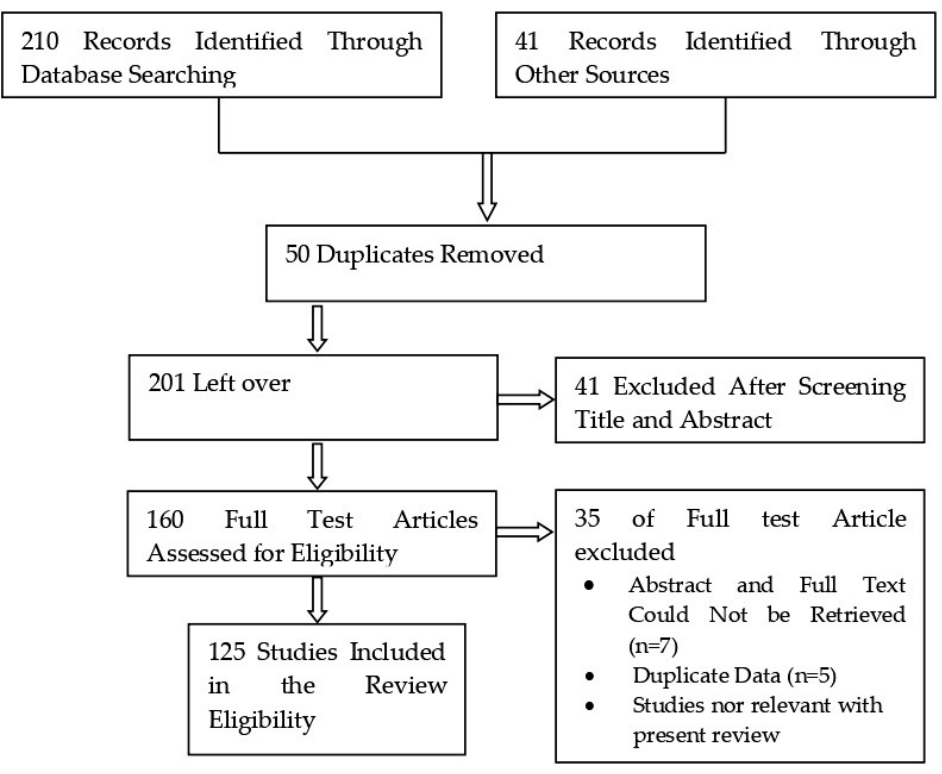

Figure 1. Flow diagram of research methodology.

\section{Botanical Description}

\subsection{Habitat}

M. esculenta is a small, evergreen, dioecious tree [7]. It is native to Republic of India and usually available in the mountain ranges from Ravi eastward to Assam, as well as Arunachal Pradesh, Meghalaya, Sikkim, Assam, Nagaland, Manipur, Mizoram in Khasi, Jaintia, Kamarupan and the Lushai hills (Figure 2) at an elevation of 900-2100 m [26,38-43]. This species is additionally found in Nepal [44,45], China [6], Vietnam [46], Sri Lanka [47], Sylhet (Bangladesh), Pakistan and Japan, Asian country islands, Himalayas [48-50] and the hills of Burma [3,5]. 


\subsection{Morphologicaland Microscopical Characteristics}

Morphological characterization of M. esculenta plant and its parts (Figure 3a-d) describes that it is a small moderate sized evergreen woody tree with a height of 3-15 m. Its leaves are lanceolate, obovate, with diameter $9 \times 3 \mathrm{~cm}$, and lower surface shows light green; upper surface dark green in appearance $[39,41]$.

Transverse sections of the leaf showed that the upper and lower epidermis consist of single-layered polygonal cells that cover the mucilaginous cuticle; vein islet and vein termination were 9-11 and 13-15, respectively [21,51]. Transverse sections of matured stem bark revealed multi-layered cork, made of rectangular, tangentially elongated, thin-walled cells, whereas the secondary cortex contained rectangular-polygonal parenchymatous cells with oval shaped starch grains [38,39,52-54].

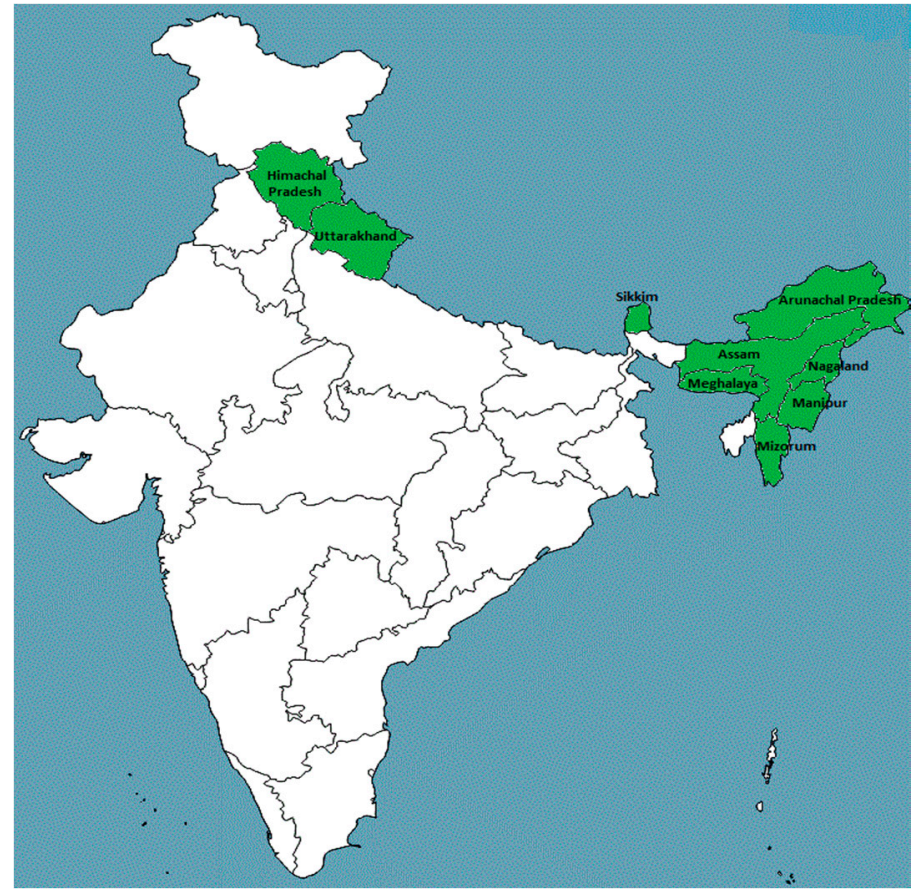

Figure 2. Natural distribution of Myrica esculenta. The shaded area represents the natural habitat of M. esculenta in the India.

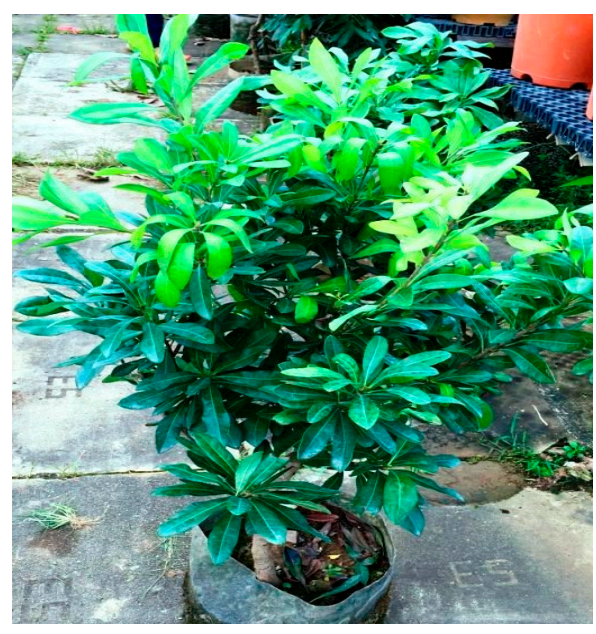

(a)

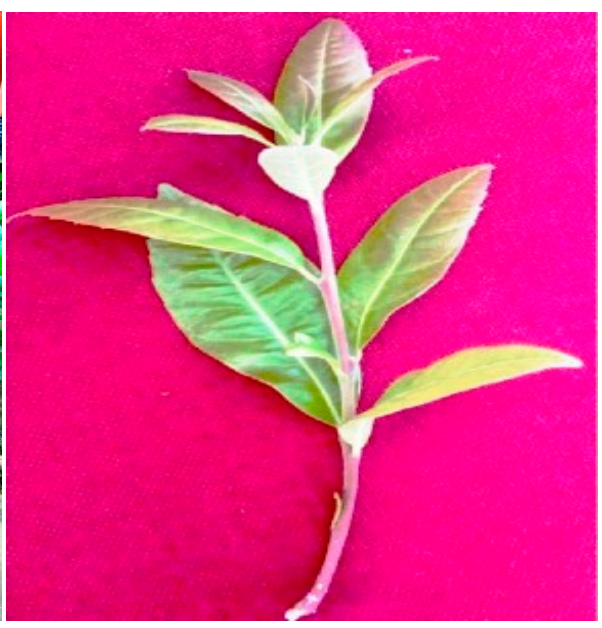

(b)

Figure 3. Cont. 


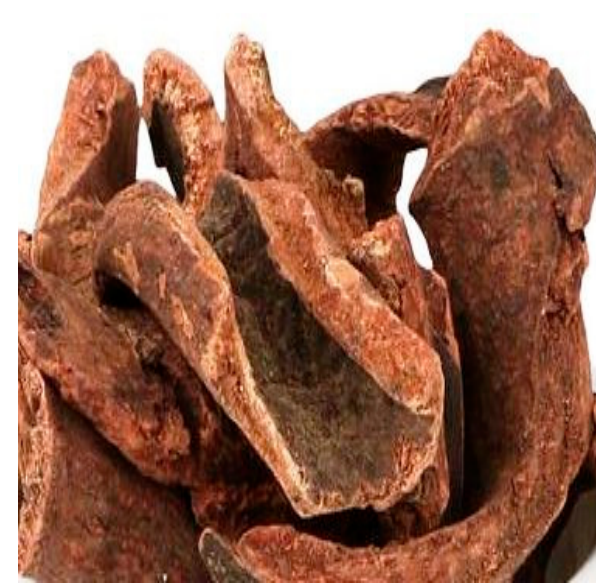

(c)

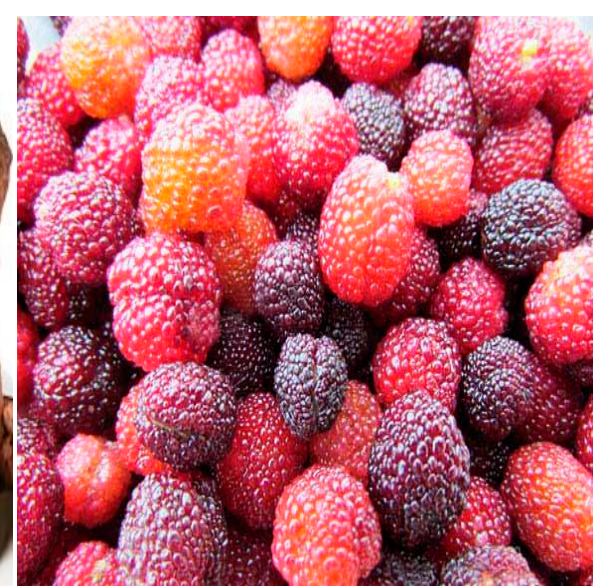

(d)

Figure 3. Myrica esculenta (a) Whole plant; (b) Leaf; (c) Bark; (d) Fruit.

\section{Ethnomedicinal Uses}

M. esculenta, a conventional ayurvedic plant, is used by different native population groups in multiple ways because of the various therapeutic uses of its bark, roots, fruits, leaves and flowers (Table 2) $[20,49,55,56]$.

Table 2. Ethomedicinal uses of M. esculenta.

\begin{tabular}{|c|c|c|c|}
\hline Plant Part Used & Uses & Region/Tribe & References \\
\hline Leaf, fruit, root, bark & Jaundice & Meghalaya, India & [23] \\
\hline Leaf & Inflammation of vocal cord & Meghalaya, India & [24] \\
\hline Bark & Antiseptic & $\begin{array}{l}\text { Meghalaya, India } \\
\text { Khasi tribe }\end{array}$ & [24] \\
\hline Fruit, bark, leaf & Fever & $\begin{array}{l}\text { Meghalaya, India } \\
\text { Vietnam, South China }\end{array}$ & [24] \\
\hline Bark & Anemia & $\begin{array}{l}\text { Meghalaya, India } \\
\text { Khasi tribe }\end{array}$ & [24] \\
\hline Fruit & $\begin{array}{l}\text { Refreshing drink "Um } \\
\text { Soh-Phi" }\end{array}$ & $\begin{array}{l}\text { Meghalaya, India } \\
\text { Khasi tribe }\end{array}$ & [24] \\
\hline Bark & Sore & $\begin{array}{l}\text { Nagaland, India } \\
\text { Zeliang tribe } \\
\text { Meghalaya, India }\end{array}$ & [43] \\
\hline Bark & Toothache & $\begin{array}{l}\text { Khasi tribe } \\
\text { Almora, Uttarakhand, India }\end{array}$ & {$[24,41,57]$} \\
\hline Bark & Sprain & $\begin{array}{c}\text { Far-flung village, Jajarkot, Nepal } \\
\text { Meghalaya, India }\end{array}$ & [58] \\
\hline Flower, bark, leaf & Inflammation, paralysis & $\begin{array}{c}\text { Khasi tribe } \\
\text { Vietnam, South China }\end{array}$ & {$[24,59]$} \\
\hline Unripe fruit & Anthelmintic & Himachal Pradesh, India & [59] \\
\hline Fruit & Bronchitis, dysentery & Nepalese community, Nepal & [60] \\
\hline Bark & Mental illness & Orissa, India & [61] \\
\hline Bark & Skin disorder & Vietnam, South China & [62] \\
\hline Bark & Cholera & Mizoram, India & {$[63,64]$} \\
\hline Bark & Cardiac debility, cardiac edema & Meghalaya, India & [64] \\
\hline
\end{tabular}


Table 2. Cont

\begin{tabular}{|c|c|c|c|}
\hline Plant Part Used & Uses & Region/Tribe & References \\
\hline Bark & Carminative & $\begin{array}{l}\text { Meghalaya, India } \\
\text { Khasi tribe } \\
\text { Mizoram, India }\end{array}$ & {$[22,64]$} \\
\hline Bark, leaf & $\begin{array}{l}\text { Asthma, chronic bronchitis, } \\
\text { lung infection }\end{array}$ & $\begin{array}{c}\text { Meghalaya, India } \\
\text { Khasi tribe } \\
\text { Vietnam, South China } \\
\text { Chaubas and Syabru, Nepal } \\
\text { Meghalaya, India }\end{array}$ & {$[23,63,65]$} \\
\hline Flower & Earache & $\begin{array}{c}\text { Khasi tribe } \\
\text { Almor, Uttarakhand, India } \\
\text { Himachal Pradesh, India } \\
\text { Meghalava, India }\end{array}$ & {$[24,41,49,66]$} \\
\hline Bark, flower, leaf, fruit & $\begin{array}{l}\text { Diarrhea, dysentery, stomach } \\
\text { problem }\end{array}$ & $\begin{array}{c}\text { Khasi tribe } \\
\text { Almora, Uttarakhand, India } \\
\text { Chungtia village, Nagaland, } \\
\text { India }\end{array}$ & {$[24,66,67]$} \\
\hline Leaf & Redness of mucosa & $\begin{array}{c}\text { Chungtia village, Nagaland, } \\
\text { India }\end{array}$ & [67] \\
\hline Fruit & Body ache & $\begin{array}{l}\text { Ukhimath block, Uttarakhand, } \\
\text { India }\end{array}$ & [68] \\
\hline Bark, fruit & Headache & $\begin{array}{c}\text { Mizoram, India } \\
\text { Ukhimath block, Uttarakhand. } \\
\text { India }\end{array}$ & {$[64,68]$} \\
\hline Fruit & Ulcer & Himalaya, India & [69] \\
\hline
\end{tabular}

Apart from these ethnomedicinal uses, various fruit industries in Himalaya used its fruits for making syrup, jam, and squash [70]. The Khasi tribe of Meghalaya uses its bark as fish poison while the extracted tannin from its bark is use as a tanning and dyeing agent [71]. Local peoples in Arunachal Pradesh use this tree for timber and fuel [22].

\section{Physiochemical and Nutritional Analysis}

Numerous physiochemical and nutritional parameters of M. esculenta have been studied, as shown in Tables 3 and 4 [22,72-74].

Table 3. Physiochemical parameters of different parts of $M$. esculenta.

\begin{tabular}{|c|c|c|c|c|c|}
\hline \multirow{2}{*}{ Parameters } & \multicolumn{4}{|c|}{ Results } & \multirow{2}{*}{ References } \\
\hline & Leaves & Bark & Stem Bark & Small Branches & \\
\hline \multicolumn{6}{|l|}{ Extractive value (\%w/w) } \\
\hline Methanolic extract & 28.32 & 38.52 & 23.57 & 5.03 & \multirow{3}{*}[8,21,55]{} \\
\hline Ethyl acetate extract & 25.46 & 21.20 & NR & NR & \\
\hline Aqueous extract & 21.28 & 15.7 & 18.36 & 3.52 & \\
\hline \multicolumn{5}{|l|}{ Ash Values (\%w/w) } & \multirow{5}{*}[8,21,55]{} \\
\hline Total ash & 2.83 & 3.3312 & 1.010 & 1.856 & \\
\hline Acid insoluble ash & 0.52 & 1.2300 & 0.187 & 0.320 & \\
\hline Foreign matter $(\% \mathrm{w} / \mathrm{w})$ & $<1 \%$ & NR & Nil & Nil & \\
\hline Loss on drying $(\% \mathrm{w} / \mathrm{w})$ & & 5 & 6.47 & 6.81 & \\
\hline Total phenolics mg of GAE/g d.w. & NR & NR & $276.78 \pm 5.36$ & $31.24 \pm 2.57$ & [8] \\
\hline Total flavonoids mg of QE/g d.w. & NR & NR & $121.68 \pm 6.81$ & $12.94 \pm 1.12$ & [8] \\
\hline
\end{tabular}


Table 4. Mineral analyses of M. esculenta fruits and stem bark.

\begin{tabular}{cccc}
\hline Minerals $(\mathbf{m g} / \mathbf{g})$ & Fruit & Stem Bark & Reference \\
\hline Calcium & $4.63 \pm 0.06$ & $3.155 \pm 0.18$ & {$[72-74]$} \\
Potassium & $7.75 \pm 0.11$ & $2.939 \pm 0.23$ & {$[72-74]$} \\
Magnesium & $8.4 \pm 0.20$ & $1.061 \pm 0.4$ & {$[72,74]$} \\
Sodium & $0.81 \pm 0.013$ & $0.060 \pm 0.03$ & {$[72,74]$} \\
Phosphorous & $0.24 \pm 0.25$ & $0.030 \pm 0.01$ & {$[73,74]$} \\
Manganese & $0.032 \pm 0.0001$ & $\mathrm{NR}$ & {$[72]$} \\
Iron & $0.404 \pm 0.0021$ & $0.123 \pm 0.16$ & {$[72,73]$} \\
Zinc & $0.216 \pm 0.0016$ & $0.006 \pm 0.001$ & {$[72,73]$} \\
Copper & $0.004 \pm 0.0002$ & $\mathrm{NR}$ & {$[72]$} \\
Sulphur & $\mathrm{NR}$ & $0.277 \pm 0.34$ & {$[73]$} \\
\hline
\end{tabular}

\section{Phytochemistry}

Phytochemical screening performed on leaves, stem bark, bark, fruits and fine branches of M. esculenta revealed several active phytoconstituents such as tannins, phenolic acids, flavonoids, terpenes, glycosides, steroids, volatile oils, and amino acids $[8,21]$. These phytoconstituents have shown a wide variety of pharmacological effects. HPTLC profiles of various extracts from different M. esculenta plant parts are presented in Table 5. The mobile phase used to develop the HPTLC chromatogram for $n$-hexane, ethyl acetate and ethanol extracts of stem bark and fine branches were toluene: ethyl acetate $(5: 5 v / v)$, toluene: ethyl acetate $(7: 3 v / v)$ and toluene: ethyl acetate: formic acid $(5: 5: 0.5 v / v)$ [8] respectively, while for leaves, ethyl acetate, methanol and aqueous extracts of leaves toluene: ethyl acetate (7:3) was used [21].

Table 5. HPTLC profile of various extracts of different parts of M. esculenta.

\begin{tabular}{|c|c|c|c|c|c|}
\hline Extract & $\begin{array}{l}\text { Wavelength } \\
\text { (nm) }\end{array}$ & Stem Bark & $\begin{array}{c}\text { Rf Value } \\
\text { Small Branches }\end{array}$ & Leaves & References \\
\hline \multirow[b]{2}{*}{ n-hexane } & 254 & $0.49,0.69,0.88$ & $0.49,0.78$ & \multirow{2}{*}{ NR } & \multirow{2}{*}{ [8] } \\
\hline & 366 & $\begin{array}{c}0.42,0.51,0.59 \\
0.74,0.83,0.91\end{array}$ & $\begin{array}{c}0.42,0.51 \\
0.74,0.83,0.91\end{array}$ & & \\
\hline \multirow[b]{2}{*}{ Ethyl acetate } & 254 & $\begin{array}{c}0.07,0.12,0.36 \\
0.47,0.61,0.67 \\
0.84\end{array}$ & $0.47,0.67$ & $0.15,0.6,0.8$ & \multirow[b]{2}{*}[8,21]{} \\
\hline & 366 & $\begin{array}{c}0.11,0.15,0.18, \\
0.33,0.38,0.55 \\
0.49,0.65,0.75 \\
\quad 0.85,0.90\end{array}$ & $\begin{array}{l}0.18,0.30,0.49,0.65 \\
0.75,0.85,0.90\end{array}$ & $\begin{array}{l}0.11,0.22,0.38 \\
0.53,0.69,0.82 \\
0.93\end{array}$ & \\
\hline \multirow{2}{*}{ Ethanol } & 254 & $0.23,0.54$ & $0.23,0.54$ & \multirow{2}{*}{ NR } & \multirow{2}{*}{ [8] } \\
\hline & 366 & $0.54,0.73,0.84$ & $\begin{array}{c}0.25,0.45,0.54,0.73 \\
0.84\end{array}$ & & \\
\hline \multirow{2}{*}{ Methanol } & 254 & \multirow{2}{*}{ NR } & \multirow{2}{*}{ NR } & $0.625,0.875$ & \multirow{2}{*}{ [21] } \\
\hline & 366 & & & $\begin{array}{c}0.46,0.58,0.81 \\
0.86,0.93\end{array}$ & \\
\hline \multirow{2}{*}{ Aqueous } & 254 & \multirow{2}{*}{ NR } & \multirow{2}{*}{ NR } & $0.1,0.63$ & \multirow{2}{*}{ [21] } \\
\hline & 366 & & & $0.093,0.65,0.81$ & \\
\hline
\end{tabular}

\subsection{Tannins and Phenolic Acids}

M. esculenta bark present gallic acid; epigallocatechin 3-O-gallate; epigallocatechin- $(4 \beta \rightarrow 8)-$ epigallocatechin3-O-gallate;3-O-galloyl-epigallocatechin- $(4 \beta \rightarrow 8)$-epigalloc-atechin3-O-gallate along with the hydrolyzable tannin castalagin [6,75]. Reversed-phase high-performance liquid chromatography analysis of fruit extract showed the presence of catechin;gallic acid; chlorogenic acid and $\rho$-coumaric acids [76]. Ethyl- $\beta$-D-glucopyranoside; 3-hydroxybenzaldehyde; isovanillin; 4-(hydroxymethyl)-phenol; 4-methoxybenzoic acid have been identified in leaves [77]. LC-MS analysis 
of fruit extract also indicated the presence of bioactive compounds, such as gallic acid and ferulic acids [78].

\subsection{Flavonoids}

Myricetin was also reported in leaves, fruits, and stem bark $[8,46,56]$, whereas quercetin was found only in leaves [79].

Two flavonoid glycosides flavone $4^{\prime}$-hydroxy-3',5,5'-trimethoxy-7-O- $\beta$-D-glucopyranosyl $(1 \rightarrow 4)$ - $\alpha$-L-rhamnopyranoside and flavone $3^{\prime}, 4^{\prime}$-dihydroxy-6-methoxy-7-O- $\alpha$-L-rhamnopyranoside were found in the leaves [79], while myricetin-3-O-(2"'-Ogalloyl)- $\alpha$-L-rhamnopyranoside and myricetin 3-O-(2" -O-galloyl)- $\alpha$-L-rhamnopyranoside were revealed in bark [78]. Myricetin 3-O-rhamnoside (myricitrin) was accounted in both M. esculenta bark, and leaves $[46,77,79,80]$.

\subsection{Terpenes}

\section{Monoterpenoid}

Myresculoside (4-hydroxy-1,8-cineole 4-O- $\beta$-dapiofuranosyl ( $1 \rightarrow 6)-\beta$-D-glucopyranoside) were reported in the leaves of M. esculenta [46].

\subsection{Triterpenoids}

Numerous triterpenoids such as lupeol; Oleanolic acid;trihydroxytaraxaranoic acid; dihydroxytaraxerane; dihydroxytaraxaranoic acid; tetrahydroxytaraxenoic aci; 3-epi-ursonic acid; arjunolic acid were reported in bark and leaves of $M$. esculenta $[46,75,81,82]$.

\subsection{Volatile Compounds}

The volatile compounds identified in leaves [83] were nerolidol; $\alpha$-pinene; $\alpha$-selinene; $\beta$-caryophyllene; $\beta$-selinen; $\alpha$-caryophyllene; $\alpha$-cadinol; linalool; whereas in bark were n-hexadecanol; eudesmol acetate and n-octadecanol [82].

\subsection{Proanthocyanidins}

M. esculenta bark revealed the presence of proanthocyanidins, such as proanthocyanidin acetate; proanthocyanidin methyl-ether and prodelhinidin $[84,85]$.

\subsection{Diarylheptanoids}

M. esculenta bark, leaves and root exhibited the presence of diaylheptanoids. Myricanol and myricnone were reported in bark $[6,84,86]$ and leaves, whereas 13 -oxomyricanolwas reported in root [86], 5-O- $\beta$-D-glucopyranosylmyricanol was accounted in leaves [45], and 16-bromomyricanol was identified in bark [86].

\subsection{Steroids}

$\beta$-rosasterol; daucosterol; $\beta$-sitosterol- $\beta$-D-glucopyranoside were identified in leaves $[77,80]$ where as taraxerol, stigmasterol were found in bark $[74,80,87] . \quad \beta$-sitosterol was identified in both $M$. esculenta leaves $[77,80]$ and bark $[81,88]$. Other miscellaneous compounds, such as amino acids; 1-ethyl-4-methylcyclohexane, myo-inositol, methyl-d-lyxofuranoside, 2-furancarboxyaldehyde, 2,5-furandionedihydro-3-methylene, furfural, oxirane were also reported in M. esculenta fruits [73,78].

The structures of some important bioactive phytoconstituents reported in M. esculenta plant are presented in Figure 4. 
<smiles>O=C(O)c1cc(O)c(O)c(O)c1</smiles>

(1)<smiles>O=C(O[C@H]1Cc2c(O)cc(O)cc2O[C@H]1c1cc(O)c(O)c(O)c1)c1cc(O)c(O)c(O)c1</smiles>

(2)

(ii)

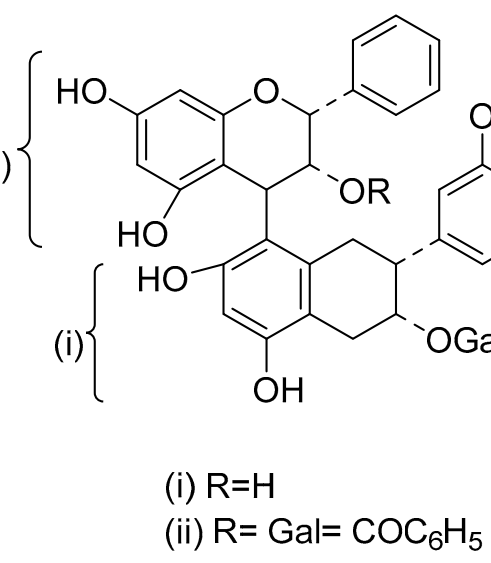

(3)

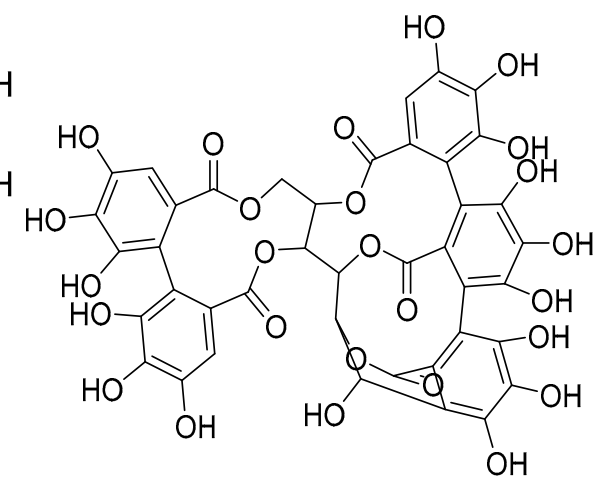

(4)<smiles>Oc1cc(O)c2c(c1)OC(c1ccc(O)c(O)c1)C(O)C2</smiles>

(5)<smiles>O=C(/C=C/c1ccc(O)c(O)c1)O[C@H]1C[C@@](O)(C(=O)O)C[C@H](O)[C@H]1O</smiles>

(6)<smiles>CCO[C@H]1O[C@H](CO)[C@@H](O)[C@H](O)[C@H]1O</smiles>

(7)

(8)

Figure 4. Cont. 
<smiles>COc1ccc(C=O)cc1O</smiles>

(9)

(10)

(11)<smiles>[Y]c1cc(-c2cc(=O)c3c(N)c(C)c(O)cc3o2)cc(O)c1N</smiles>

(12)

(13)
$\mathrm{R}_{1} \quad \mathrm{R}_{2}$
$\mathrm{R}_{3}$
$\mathrm{R}_{4} \quad \mathrm{R}_{5} \quad \mathrm{R}_{6}$

(a) $\mathrm{OCH}_{3} \quad \mathrm{H} \quad \beta$-Dglucopyranosyl-(1-4)- $\alpha-\mathrm{L} \quad \mathrm{OCH}_{3} \quad \mathrm{OH} \quad \mathrm{OCH}_{3}$

(b) $\quad \mathrm{H} \quad \mathrm{OCH}_{3} \quad \alpha$-L-rhamnopyranoside $\quad \mathrm{OH} \quad \mathrm{OH} \quad \mathrm{H}$<smiles>C[C@@H]1OC(Oc2c(-c3cc(O)c(O)c(O)c3)oc3cc(O)cc(O)c3c2=O)[C@H](O)[C@@H](O)[C@H]1O</smiles>

(14)<smiles>CC1(C)CCC2(C(=O)O)CC[C@H]3C(=CCC4[C@@]5(C)CC[C@@H](O)C(C)(C)C5CC[C@]43C)C2C1</smiles>

(16)

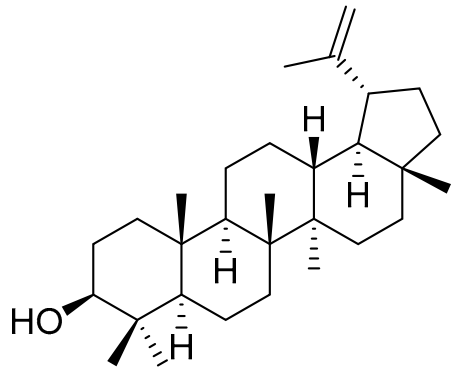

(15)

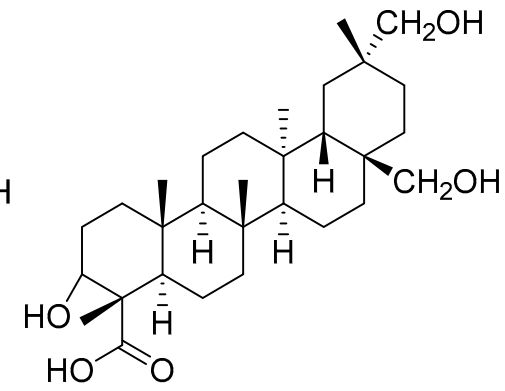

(17)

Figure 4. Cont. 


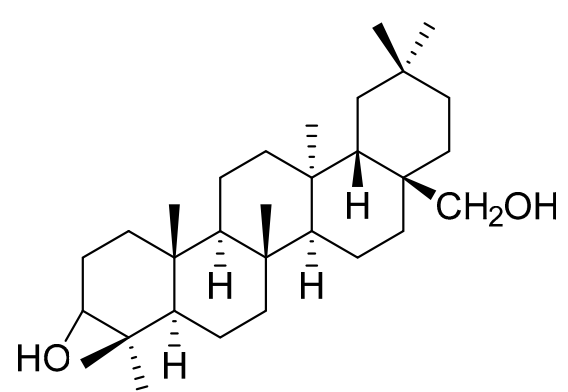

(18)

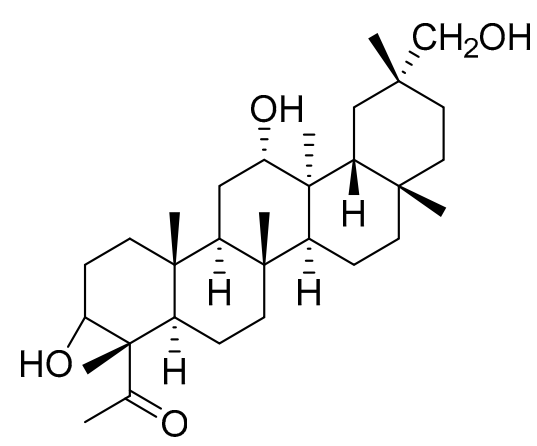

(20)<smiles>Cc1cc(O)c(C2CC(c3ccc(O)c(O)c3)Oc3cccc(O)c32)c2c1CC(O)C(c1ccc(O)c(O)c1)C2</smiles>

(22)

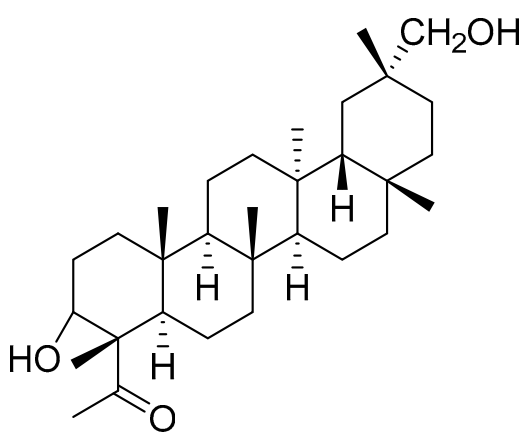

(19)<smiles>C[C@H]1CC[C@]2(O)CC[C@]3(C)C(=CCC4[C@@]5(C)CC[C@H](O)C(C)(C)C5CC[C@]43C)C2[C@@H]1C</smiles>

(21)<smiles>COc1ccccc1-c1cc(CCC[C@@H](O)CCCCc2ccc(O)c(OC)c2OC)ccc1O</smiles>

(23)<smiles></smiles>

(24)

Figure 4. Structure of some isolated bioactive compounds from different parts of M. esculenta. (1) Gallic acid, (2) Epigallocatechin 3-O-gallate, (3) i) Epigallocatechin- $(4 \beta \rightarrow 8)$-epigallocatechin-3-O-gallate, ii) 3-O-galloyl-epigallocatechin- $(4 \beta \rightarrow 8)$-epigallocatechin-3-O-gallate, (4) Castalagin, (5) Catechin, (6) Chlorogenic acid, (7) $p$-coumaric acid, (8) Ethyl- $\beta$-D-glucopyranoside, (9) 3-hydroxybenzaldehyde, (10) Isovanillin, (11) Ferulic acid, (12) Myricetin, (13) i) Flavone $4^{\prime}$-hydroxy-3',5,5'-trimethoxy-7-O- $\beta$-D-glucopyranosyl $(1 \rightarrow 4)$ - $\alpha$-L-rhamnopyranoside, ii) flavone $3^{\prime}, 4^{\prime}$-dihydroxy-6-methoxy-7-O- $\alpha$-L-rhamnopyranoside, (14) Myricitrin, (15) Lupeol, (16) Oleanolic acid, (17) Trihydroxytaraxaranoic acid, (18) Dihydroxytaraxerane, (19) Dihydroxytaraxaranoic acid, (20) Tetrahydroxytaraxenoic acid, (21) 3-epi-ursonic acid, (22) Prodelphinidin dimer, (23) Myricanol, (24) Myricanone. 


\section{Pharmacological Profile}

Extracts from M. esculenta possess a broad spectrum of pharmacological activities. Previous research revealed that phenolic compounds are highly active antioxidants, and such antioxidant-rich botanicals offer promising potential in the management of degenerative ailments. Phenolic compounds are secondary metabolites synthesized in plants in response to environmental stresses such as attacks from pathogens and insects, UV radiation, and injuries [5-7]. These phytochemicals have the ability to eliminate hydroxyl radicals [89], superoxide anion radicals [90], lipid peroxyl radicals [91] and even to chelate metals, besides to play a vital role in the stability of food products, as well as in the defense mechanisms of biological systems $[4,8]$. These molecules also prevent oxidative losses and have cytoprotective, anti-inflammatory, and adaptogenic properties. It was found that relatively high amounts of phenolic compounds are present in M. esculenta fruits than M. rubra [76]. The antioxidant activity of M. esculenta fruits and bark has been reported by using different antioxidant assays.

Previous research confirmed that presence of phenolic acids and flavonoids is responsible for its antioxidant potential [78,92-98]. But other pharmacological activities have been also reported, including analgesic [50,92,93], antiasthmatic [98-102], anticancer [78,103], antidepressant [61,104], antidiabetic [105], antidiarrheal [106], anthelmintic [106,107], antihypertensive [45], anti-inflammatory [50,94,108], antimicrobial [73,78,109-111], antipyretic [93], antiulcer [112], anxiolytic [61], chemopreventive [113], hepatoprotective [114], wound healing [59], and non-toxicity [105] effects. Simultaneously, several in vitro and in vivo studies on pharmacological profile of $M$. esculenta are under way. Scientific exploration has revealed that different types of M. esculenta extracts possess multiple bioactive attributes (Table 6).

Table 6. Biological effects of M. esculenta.

\begin{tabular}{|c|c|c|c|c|c|c|}
\hline Part Use & Extract/Fraction & $\begin{array}{l}\text { Dose Tested/Route of } \\
\text { Administration }\end{array}$ & $\begin{array}{l}\text { Animals/Cell } \\
\text { Lines }\end{array}$ & Experimental Models & Result & Reference \\
\hline \multicolumn{7}{|c|}{ Anti-inflammatory } \\
\hline Leaves & Methanolic & 200 mg/kg, p.o. & Rat & $\begin{array}{l}\text { Carrageenan-induced } \\
\text { rat paw edema }\end{array}$ & $\begin{array}{c}\text { Significant } \\
\text { anti-inflammatory } \\
\text { activity }\end{array}$ & [93] \\
\hline Stem Bark & Essential oil & $10 \mathrm{~mL}$ per ear & $\begin{array}{l}\text { Swiss albino } \\
\text { mice }\end{array}$ & In vitro [ear] & $\begin{array}{c}\text { Significant } \\
\text { anti-inflammatory } \\
\text { potential }\end{array}$ & [111] \\
\hline Leaves & ME- EtAC & $\begin{array}{c}100,200 \text { and } 400 \\
\mathrm{mg} / \mathrm{kg} \text {, p.o. }\end{array}$ & Wistar rats & $\begin{array}{l}\text { Carrageenan-induced } \\
\text { rat paw edema }\end{array}$ & $\begin{array}{c}\text { Significant } \\
\text { anti-inflammatory } \\
\text { activity }\end{array}$ & [50] \\
\hline Bark & $\begin{array}{l}\text { Ethyl acetate and } \\
\text { aqueous }\end{array}$ & $\begin{array}{c}100 \text { and } 200 \mathrm{mg} / \mathrm{kg} \text {, } \\
\text { p.o. }\end{array}$ & $\begin{array}{l}\text { Wistar } \\
\text { albino rats }\end{array}$ & $\begin{array}{c}\text { Carrageenan and } \\
\text { histamine induced rat } \\
\text { paw edema }\end{array}$ & $\begin{array}{c}\text { Significant } \\
\text { anti-inflammatory } \\
\text { potential } \\
(\mathrm{EAE}>\mathrm{AE})\end{array}$ & [102] \\
\hline \multicolumn{7}{|c|}{ Antimicrobial } \\
\hline Stem bark & Volatile oil & $10 \mathrm{~mL}$ & $\begin{array}{c}\text { BP, SA, SE, } \\
\text { EC, PA, CA, } \\
\text { AN and SC }\end{array}$ & - & $\begin{array}{c}\text { Significant } \\
\text { antimicrobial } \\
\text { potential }\end{array}$ & [111] \\
\hline $\begin{array}{l}\text { Bark and } \\
\text { fruit }\end{array}$ & $\begin{array}{l}\text { Methanolic and } \\
\text { chloroform }\end{array}$ & - & - & $\begin{array}{l}\text { Agar Well diffusion } \\
\text { method }\end{array}$ & $\begin{array}{c}\text { Significant } \\
\text { antimicrobial } \\
\text { potential (Bark> } \\
\text { Fruits) }\end{array}$ & [109] \\
\hline Fruit pulp & Ethanolic & 10 and $50 \mathrm{mg} / \mathrm{ml}$ & In vitro & Disc diffusion assay & $\begin{array}{c}\text { Dose dependent } \\
\text { antimicrobial } \\
\text { potential }\end{array}$ & [73] \\
\hline Fruit & Methanolic & $50 \mu \mathrm{L}$ & $\begin{array}{l}\text { SA, SE, BS, } \\
\text { PM, EC, SE }\end{array}$ & $\begin{array}{l}\text { Agar Well diffusion } \\
\text { method }\end{array}$ & $\begin{array}{c}\text { Significant } \\
\text { potential against } \\
\text { Pathogens }\end{array}$ & \\
\hline \multicolumn{7}{|c|}{ Antifungal } \\
\hline Fruit & $\begin{array}{l}\text { Methanolic, } \\
\text { ethanolic and } \\
\text { aqueous }\end{array}$ & 10 and $50 \mathrm{mg} / \mathrm{ml}$ & $\begin{array}{l}\text { Candida } \\
\text { albicans, } \\
\text { Aspergillus } \\
\text { flavus and } \\
\text { Aspergillus } \\
\text { parasiticus }\end{array}$ & Disc diffusion assay & $\begin{array}{l}\text { Significant } \\
\text { antifungal } \\
\text { activity }\end{array}$ & [73] \\
\hline
\end{tabular}


Table 6. Cont

\begin{tabular}{|c|c|c|c|c|c|c|}
\hline Part Use & Extract/Fraction & $\begin{array}{l}\text { Dose Tested/Route of } \\
\text { Administration }\end{array}$ & $\begin{array}{l}\text { Animals/Cell } \\
\text { Lines }\end{array}$ & Experimental Models & Result & Reference \\
\hline \multicolumn{7}{|c|}{ Anthelmintic } \\
\hline Bark & $\begin{array}{l}50 \% \text { Aqueous } \\
\text { Ethanolic }\end{array}$ & $12.5,25$ and $50 \mathrm{mg} / \mathrm{ml}$ & $\begin{array}{c}\text { Earthworms } \\
\text { (Pheretima } \\
\text { posthuma) }\end{array}$ & - & $\begin{array}{c}\text { Paralysis and } \\
\text { death at } 12.5 \\
\text { mg.ml }\end{array}$ & [107] \\
\hline \multicolumn{7}{|c|}{ Anticancer } \\
\hline Fruit & $\begin{array}{l}\text { Methanol, acid } \\
\text { methanol acetone } \\
\text { and acidic acetone }\end{array}$ & $\begin{array}{l}66.7,166.5,333,500 \\
667 \mu \mathrm{g} \text { f.w. } / 100 \mu \mathrm{L} \\
\text { culture medium }\end{array}$ & $\begin{array}{l}\mathrm{C}_{33} \mathrm{~A}, \mathrm{SiHa} \\
\text { and HeLa } \\
\text { cell lines }\end{array}$ & - & $\begin{array}{l}\text { Acetone and } \\
\text { acidic acetone } \\
\text { extracts showed } \\
\text { anticancer } \\
\text { potential }\end{array}$ & [108] \\
\hline Fruit & Methanolic & $5 \mathrm{mg} / \mathrm{ml}$ & $\begin{array}{l}\text { HepG2, Hela } \\
\text { and } \\
\text { MDA-MB-231 } \\
\text { cells }\end{array}$ & MTT assay & $\begin{array}{l}\text { Moderate } \\
\text { anticancer } \\
\text { activity }\end{array}$ & [78] \\
\hline \multicolumn{7}{|c|}{ Chemopreventive } \\
\hline Bark & Ethanolic & $2.0 \mathrm{mg}$ and $4.0 \mathrm{mg} / \mathrm{kg}$ & $\begin{array}{l}\text { Swiss albino } \\
\text { mice }\end{array}$ & $\begin{array}{c}\text { Cumene } \\
\text { hydroperoxide-mediated } \\
\text { cutaneous oxidative } \\
\text { stress and toxicity }\end{array}$ & $\begin{array}{c}\uparrow \text { antioxidant } \\
\text { enzymes activity }\end{array}$ & [113] \\
\hline \multicolumn{7}{|c|}{ Antioxidant } \\
\hline Fruit & Methanolic & - & In vitro & $\begin{array}{l}\text { DPPH, ABTS and } \\
\text { FRAP assay }\end{array}$ & $\begin{array}{l}\text { Significant } \\
\text { antioxidant } \\
\text { activity }\end{array}$ & [78] \\
\hline Fruit pulp & Methanolic & $0.10 \mathrm{ml}$ & In vitro & $\begin{array}{l}\text { DPPH, ABTS and } \\
\text { FRAP assay }\end{array}$ & $\begin{array}{l}\text { Good scavenging } \\
\text { potential }\end{array}$ & [76] \\
\hline Fruit & $\begin{array}{l}\text { Aqueous methanol } \\
\text { and acetone }\end{array}$ & $100 \mu \mathrm{l}$ & In vitro & DPPH assay & $\begin{array}{l}\text { Acetone extract } \\
\text { showed higher } \\
\text { scavenging } \\
\text { potential }\end{array}$ & [94] \\
\hline Fruit & $\begin{array}{l}\text { Methanol, acidic } \\
\text { methanol, acetone, } \\
\text { and acidic-acetone }\end{array}$ & - & In vitro & $\begin{array}{c}\text { DPPH, ABTS, FRAP } \\
\text { and Superoxide anion } \\
\text { radicals scavenging } \\
\text { assay }\end{array}$ & $\begin{array}{l}\text { MeAA showed } \\
\text { higher } \\
\text { antimicrobial } \\
\text { potential and } \\
\text { MeAM and MeA } \\
\text { intermediate }\end{array}$ & [103] \\
\hline Fruit & Fruit Juice & $0.2-2.0 \mathrm{mg} / \mathrm{mL}$ & In vitro & $\begin{array}{c}\mathrm{DPPH}, \mathrm{H}_{2} \mathrm{O}_{2} \text { and NO } \\
\text { scavenging activity }\end{array}$ & $\begin{array}{l}\text { Moderate } \\
\text { antioxidant } \\
\text { activity }\end{array}$ & [95] \\
\hline \multicolumn{7}{|c|}{ Antidiabetic } \\
\hline Leaves & Methanolic & $\begin{array}{c}50,100 \text { and } 150 \mathrm{mg} / \mathrm{kg} \text {, } \\
\text { p.o. }\end{array}$ & $\begin{array}{c}\text { Albino } \\
\text { wistar rats }\end{array}$ & STZ induced diabetes & $\begin{array}{c}\text { Significant } \\
\text { anti-dyslipidemic } \\
\text { effect at } 150 \\
\mathrm{mg} / \mathrm{kg} \text { and } \\
\text { maintain blood } \\
\text { glucose level }\end{array}$ & [105] \\
\hline \multicolumn{7}{|c|}{ Hepatoprotective } \\
\hline $\begin{array}{l}\text { Polyherbal } \\
\text { formulation } \\
\text { (Herbitars) }\end{array}$ & - & 50 and $100 \mathrm{mg} / \mathrm{kg}$ & Wistar rat & $\begin{array}{l}\mathrm{CCl}_{4} \text { induced } \\
\text { hepatotoxicity }\end{array}$ & $\begin{array}{l}\text { Extract } \downarrow \text { TBARS, } \\
\uparrow S O D, C A T, \text { GSH }\end{array}$ & [114] \\
\hline \multicolumn{7}{|c|}{ Antidepressant } \\
\hline Bark & Methanolic & $300,500 \mathrm{mg} / \mathrm{kg}$, p.o. & Albino mice & $\begin{array}{c}\text { Open field test, } \\
\text { cage-crossing test, } \\
\text { head-dip test, rearing } \\
\text { test, traction test, forced } \\
\text { swimming test }\end{array}$ & $\begin{array}{l}\text { Significant } \\
\text { antidepressant } \\
\text { activity }\end{array}$ & [104] \\
\hline \multicolumn{7}{|c|}{ Anxiolytic } \\
\hline Bark & Ethanolic & $\begin{array}{l}100,200 \text { and } 400 \\
\mathrm{mg} / \mathrm{kg}\end{array}$ & Rats & $\begin{array}{l}\text { Tail suspension test } \\
\text { Forced swimming test }\end{array}$ & $\begin{array}{c}\text { Significant and } \\
\text { dose dependent } \\
\text { anxiolytic activity }\end{array}$ & [61] \\
\hline \multicolumn{7}{|c|}{ Antihypertensive } \\
\hline Leaves & Methanolic & $100 \mathrm{mM}$ & In vitro & ACE inhibitory activity & $\begin{array}{c}\text { Potent ACE } \\
\text { inhibition } \\
\text { potential }\end{array}$ & [45] \\
\hline \multicolumn{7}{|c|}{ Antiasthmatic } \\
\hline
\end{tabular}


Table 6. Cont.

\begin{tabular}{|c|c|c|c|c|c|c|}
\hline Part Use & Extract/Fraction & $\begin{array}{l}\text { Dose Tested/Route of } \\
\text { Administration }\end{array}$ & $\begin{array}{l}\text { Animals/Cell } \\
\text { Lines }\end{array}$ & Experimental Models & Result & Reference \\
\hline Bark & Ethanolic & 75 mg/kg, p.o. & Guinea pig & $\begin{array}{l}\text { Acetylcholine induced } \\
\text { bronchospasm }\end{array}$ & $\begin{array}{c}\text { Protection } \\
\text { against } \\
\text { bronchospasm } \\
\text { and anaphylaxis }\end{array}$ & [98] \\
\hline Bark & Ethanolic & 75 mg/kg, p.o. & In vitro & $\begin{array}{l}\text { Guinea pig tracheal } \\
\text { strip }\end{array}$ & $\begin{array}{l}\downarrow \mathrm{pD} 2 \text { value of } \\
\text { histamine and } \\
\text { acetylcholine }\end{array}$ & [98] \\
\hline Stem bark & Ethanolic & 150 mg/kg, p.o. & Guinea pig & $\begin{array}{l}\text { Histamine induced } \\
\text { bronchospasm }\end{array}$ & $\downarrow$ TLC and DLC & [115] \\
\hline Stem Bark & Ethanolic & 75 and $150 \mathrm{mg} / \mathrm{kg}$, p.o. & Mice & $\begin{array}{l}\text { Acetic acid induced } \\
\text { vascular permeability } \\
\text { and allergic pleurisy }\end{array}$ & & [99] \\
\hline Stem bark & Aqueous extract & 27 \& 54mg/kg p.o. & $\begin{array}{l}\text { Guinea pig } \\
\text { In vitro }\end{array}$ & $\begin{array}{l}\text { histamine induced } \\
\text { bronchospasm } \\
\text { Guinea pig tracheal } \\
\text { chain }\end{array}$ & $\begin{array}{c}\text { Significant } \\
\text { antiasthamtic } \\
\text { potential }\end{array}$ & [100] \\
\hline Bark & $\begin{array}{l}\text { Polar, non-polar } \\
\text { and methanolic }\end{array}$ & 200 mg/kg, p.o. & $\begin{array}{l}\text { Rat and } \\
\text { in vitro }\end{array}$ & $\begin{array}{l}\text { Acetylcholine induced } \\
\text { bronchospasm in } \\
\text { conscious guinea pigs; } \\
\text { acetylcholine induced } \\
\text { contraction on isolated } \\
\text { guinea pig tracheal } \\
\text { chain preparation; } \\
\text { compound } 48 / 80 \\
\text { induced mast cell } \\
\text { degranulation using rat; } \\
\text { and trypsin and egg } \\
\text { albumin induced } \\
\text { bronchospasm }\end{array}$ & $\begin{array}{c}\text { PE showed } \\
\text { higher potential } \\
\text { than NPE and } \\
\text { ME }\end{array}$ & [101] \\
\hline \multicolumn{7}{|c|}{ Antiulcer } \\
\hline Bark & Ethanolic & 100 and $200 \mathrm{mg} / \mathrm{kg}$ & Albino rat & $\begin{array}{l}\text { Pyloric ligation } \\
\text { induced ulcer }\end{array}$ & $\begin{array}{c}\downarrow l e v e l \text { of GV, FA, } \\
\text { LPO and GSH } \\
\text { and } \uparrow \text { CAT, } \\
\text { nitrate and } \\
\text { MPO } \downarrow l e v e l \text { of GV, } \\
\text { FA, LPO and } \\
\text { GSH and } \uparrow \text { CAT, } \\
\text { nitrate and MPO }\end{array}$ & [112] \\
\hline \multicolumn{7}{|c|}{ Antidiarrheal } \\
\hline Leaves & Ethanolic & $\begin{array}{l}250 \text { and } 500 \mathrm{mg} / \mathrm{kg} \text {, } \\
\text { p.o. }\end{array}$ & Mice & $\begin{array}{c}\text { Castor-oil induced } \\
\text { diarrhea }\end{array}$ & $\begin{array}{c}\text { Significant } \\
\text { antidiarrheal } \\
\text { activity } \\
\end{array}$ & [106] \\
\hline \multicolumn{7}{|c|}{ Antipruritic } \\
\hline Stem bark & Aqueous & $150 \mathrm{mg} / \mathrm{kg}$ & Male mice & $\begin{array}{l}\text { Compound } \\
\text { 48/80-induced }\end{array}$ & $\begin{array}{l}\text { Significantly } \\
\text { decrease in } \\
\text { scratching effect }\end{array}$ & [116] \\
\hline \multicolumn{7}{|c|}{ Analgesic } \\
\hline Fruit & Methanolic & $50,100 \mathrm{mg} / \mathrm{kg}$, p.o. & Mice & $\begin{array}{l}\text { Eddy's hot plate } \\
\text { method }\end{array}$ & $\begin{array}{c}\text { Significant } \\
\text { analgesic activity }\end{array}$ & [92] \\
\hline Leaves & ME-EtAC & 100,200 mg/kg, p.o. & Mice & $\begin{array}{l}\text { Acetic acid induced } \\
\text { writhing and tail } \\
\text { immersion assay }\end{array}$ & $\begin{array}{l}\text { Significant } \\
\text { response at } 200 \\
\mathrm{mg} / \mathrm{kg}\end{array}$ & {$[50]$} \\
\hline Leaves & Methanolic & 200 mg/kg, p.o. & Mice & $\begin{array}{c}\text { Acetic acid induced } \\
\text { writhing }\end{array}$ & $\begin{array}{l}54.56 \% \text { inhibition } \\
\text { of writhing }\end{array}$ & [93] \\
\hline \multicolumn{7}{|c|}{ Antipyretic } \\
\hline Fruit & Methanolic & 50 and $100 \mathrm{mg} / \mathrm{kg}$ & Mice & $\begin{array}{l}\text { Yeast induced pyrexia } \\
\text { in mice }\end{array}$ & $\begin{array}{l}\text { Significant } \\
\text { antipyretic effect } \\
\text { at } 100 \mathrm{mg} / \mathrm{kg}\end{array}$ & [92] \\
\hline \multicolumn{7}{|c|}{ Wound healing } \\
\hline Bark & Aqueous & $\begin{array}{l}\text { Ointment }(100 \\
\left.\mathrm{mg} / 500 \mathrm{~mm}^{2}\right)\end{array}$ & Albino rats & $\begin{array}{l}\text { Wound excision and } \\
\text { incision }\end{array}$ & $\begin{array}{c}\text { Significant } \\
\text { wound healing } \\
\text { potential }\end{array}$ & [59] \\
\hline
\end{tabular}

Previous studies reported that the toxic impacts of methanolic extract of M. esculenta leaves and found no indication of lethality up to the dose of $300 \mathrm{mg} / \mathrm{kg}$ by oral route for two weeks. In any case, $2000 \mathrm{mg} / \mathrm{kg}$ of lethal impact measurements of the methanol extract were seen in Wistar rats [100]. 
Furthermore, intense poisonous quality examinations performed with ethyl acetate and aqueous extracts of M. nagi bark at three different intravenous dosages $(100,200$ and $1000 \mathrm{mg} / \mathrm{kg}$ ) demonstrated that the $\mathrm{LD}_{50}$ of the ethyl acetate and aqueous extracts in mice was $1000 \mathrm{mg} / \mathrm{kg}$ [98].

\section{Conservation}

Demolition of plant assets is an ordinary event. The current rate of eradication caused by mankind is about hundreds of time faster compared to the natural rate of elimination [117]. Due to training exercises in the Himalayan district, the existence of numerous therapeutically effective botanicals, including M. esculenta, are threatened with extinction. M. esculenta is exchanged and used most often as a conventional medication. Because of its numerous uses, application is on the rise through national and worldwide exchange, leading to the expansion of wild populaces. This has brought exceptional declines in population $[118,119]$. Due to its extreme overuse from regular natural surroundings, limited geographic predominance, and uncertain inalienable issues of seed practicality and seed germination, elective methodologies for spread and protection are urgently expected to evade the potential termination of this indispensable species [8,27]. The village forest council framework is a town-level establishment, and it has impressive potential for involving local communities in forest management for conservation [119]. Biotechnology offers new methods for enhancing biodiversity and biotechnological methodologies. For example, micropropagation systems have gotten more consideration and may assume a fundamental part in the foundation of hereditarily unvarying botanicals for the business. Hopefully, the advancement of coherent micropropagation conventions could ensure satisfactory availability of the M. esculenta plant (without forced ecological imperatives) with a consequent lessening in uncontrolled collecting weight on wild populaces [27]. Likewise, there are several highly valued traditional Indian ethnomedicinal plants having rich therapeutic potential and need immense scientific exploration and conservation strategies [120-122].

\section{Conclusions and Future Perspectives}

M. esculenta has been used for its restorative and dietary potentials, from the old-fashioned Ayurveda and Unani arrangement of solution. It is clear in this review that $M$. esculenta contains various phytochemicals, which are responsible for the therapeutic estimate of this plant. M. esculenta, and have been responsible for several pharmacological impacts in the treatment of different diseases, including asthma, diabetes, tumors, ulcer, tension; however, being a rich wellspring of vitamin $C$ and polyphenolic compounds, there is a need to investigate the capacity of this plant for immunomodulatory, cardioprotective, nephroprotective, and neuroprotective movement. Although there are many analyses of chemical constituents, and the pharmacological activity has been reported for this plant, the mechanism of pharmacological action and the metabolites responsible for these activities should be studied in more detail. The population of this restorative and practical plant species is on the reverse because of excessive exploitation of woodlands and wastelands, neglect of practicable assets, poor development, and poor recovery of species in characteristic natural surroundings. Subsequently, a great opportunity has already passed to make the vital movement to expand its populace measure, efficiency, protection, and even use.

Author Contributions: All authors read and approved the final version of the manuscript.

Funding: This research received no external funding.

Acknowledgments: Authors are thankful to A.P. Singh, Dean RIC, I. K. Gujral Punjab Technical University and members of staff in the department of RIC, I. K. Gujral Punjab Technical University for support and encouragement in this work. N. Martins would like to thank the Portuguese Foundation for Science and Technology (FCT-Portugal) for the Strategic project ref. UID/BIM/04293/2013 and "NORTE2020 - Northern Regional Operational Program" (NORTE-01-0145-FEDER-000012).

Conflicts of Interest: The authors declare no conflict of interest. 


\section{Abbreviations}

The following abbreviations are used in this manuscript:

HPTLC High performance thin layer chromatography

LD50 Lethal dose 50

ME-EtAC Ethyl acetate fraction-Myrica esculenta

DPPH 2,2-diphenyl-1-picrylhydrazyl

ABTS 2,2-azinobis (3-ethyl-benzothiazoline-6-sulfonic acid)

FRAP Ferric reducing antioxidant power

MTT Methyl thiazolyl tetrazolium

BP Bacillus pumilus

SA Staphylococcus aureus

SE Staphylococcus epidermidis

EC Escherichia coli

BS Bacillus subtilis

PM Proteus mirabilis

PA Pseudomonas aeruginosa

CA Candida albicans

AN Aspergillus niger

SC Sacharomyces cerevisiae

$\mathrm{CCl}_{4} \quad$ Carbon tetrachloride

$\mathrm{H}_{2} \mathrm{O}_{2} \quad$ Hydrogen peroxide

ACE Angiotensin Converting Enzyme

$\mathrm{MeOH}$ methanol

nm Nanometer

EAE Ethyl acetate extract

ME Methanolic extract

AE Aqueous extract

PE Polar extract

NPE Non polar extract

STZ Streptozotocin

p.o. Per oral

TBARS Thio barbituric acid reactive substances

CAT Catalase

SOD Superoxide dismutase

GSH Glutathione

MPO Myeloperoxidase

GV Gastric volume

FA Free acidity

DLC Differential Leukocyte Count

TLC Total Leukocyte Count

$\mathrm{mg} / \mathrm{g} \quad$ Milligram per gram

$\mathrm{mg} / \mathrm{kg} \quad$ Milligram per kilogram

$\% \mathrm{w} / \mathrm{w} \quad$ Percentage weight by weight

GAE/g Gallic Acid Equivalent per gram

QE/g Quercetin equivalent per gram

\section{References}

1. Yanthan, M.; Misra, A.K. Molecular approach to the classification of medicinally important actinorhizal genus Myrica. Indian J. Biotechnol. 2013, 12, 133-136.

2. Silva, B.J.C.; Seca, A.M.L.; Barreto, C.M.D.; Pinto, D.C.G.A. Recent breakthroughs in the antioxidant and anti-inflammatory effects of Morella and Myrica species. Int. J. Mol. Sci. 2015, 16, 17160-17180. [CrossRef] [PubMed] 
3. Kumar, A.; Rana, A.C. Pharmacognostic and pharmacological profile of traditional medicinal plant: Myrica nagi. Int. Res. J. Pharm. 2013, 3, 32-37.

4. Sun, C.; Huang, H.; Xu, C.; Li, X.; Chen, K. Biological activities of extracts from Chinese bayberry (Myrica rubra Sieb. et Zucc.): A review. Plant Foods Hum. Nutr. 2013, 68, 97-106. [CrossRef] [PubMed]

5. Sood, P.; Shri, R. A review on ethnomedicinal, phytochemical and pharmacological aspects of Myrica esculenta. Indian J. Pharm. Sci. 2018, 80, 2-13.

6. Sun, D.; Zhao, Z.; Wong, H.; Foo, L.Y. Tannins and other phenolics from Myrica esculenta bark. Phytochemistry 1988, 27, 579-583.

7. Annonymous. The Wealth of India; Council of Scientific and Industrial Research: New Delhi, India, 1962; p. 472.

8. Srivastava, B.; Sharma, V.C.; Pant, P.; Pandey, N.K.; Jadhav, A.D. Evaluation for substitution of stem bark with small branches of Myrica esculenta for medicinal use-A comparative phytochemical study. J. Ayurveda Integr. Med. 2016, 7, 1-6. [CrossRef]

9. Nadkarni, K.M. Indian Materia Medica, 3rd ed.; Popular Book Depot: Mumbai, India, 2002; p. 871.

10. Huguet, V.; Gouy, M.; Normand, P.; Zimpfer, J.M.; Fernandez, M.P. Molecular phylogeny of Myricaceae: A reexamination of host symbiont specificity. Mol. Phylogenet. Evol. 2005, 34, 557-568. [CrossRef]

11. Bhatt, I.D.; Rawal, R.S.; Dhar, U. Improvement in seed germination of Myrica esculenta Buch. Ham. Ex D. Don- A high value tree species of Kumanun Himalaya, India. Seed Sci. Technol. 2000, 28, 597-605.

12. Catalogue of Life: 2019 Annual Checklist. Available online: http://www.catalogueoflife.org/col/search/all/ key/myrica+esculenta+/fossil/1/match/1 (accessed on 15 May 2019).

13. Pandey, G.; Sharma, B.D.; Hore, D.K.; Rao, N.K. Indigenous minor fruits genetic resources and their marketing status in north-eastern hills of India. J. Hill Res. 1993, 6, 1-4.

14. Makdoh, K.; Lynser, M.B.; Pala, K.H.M. Marketing of Indigenous Fruits: A Source of Income among Khasi Women of Meghalaya, North East India. J. Agric. Sci. 2014, 5, 1-9. [CrossRef]

15. MacDnald, A.D. The morphology and relationships of the Myricaceae. Evol. Syst. Foss. Hist. Hamamelidae 1989, 2, 147-165.

16. Haridasan, K.; Rao, R.R. Forest Flora of Meghalaya; Caprifoliaceae to Salicaceae; Bishan Singh Mahendra Pal Singh: Dehradun, India, 1987; Volume 2, pp. 851-852.

17. Paranjpe, P. Indian Medicinal Plants, 3rd ed.; Chaukhamba Sanskrit Pratishthan: New Delhi, India, 2012; p. 128.

18. Bhatt, I.D.; Dhar, U. Factors controlling micropropagation of Myrica esculenta Buch. -Ham. ex D. Don: A high value wild edible of Kumaun Himalaya. Afr. J. Biotechnol. 2004, 3, 534-540.

19. Bao, J.; Cai, Y.; Sun, M.; Wang, G.; Corke, H. Anthocyanins, flavonols, and free radical scavenging activity of Chinese Bayberry (Myrica rubra) extracts and their color properties and stability. J. Agric. Food Chem. 2005, 53, 2327-2332. [CrossRef] [PubMed]

20. The Ayurvedic Pharmacopoeia of India, part I, 1st ed.; Government of India, Ministry of Health and Family Welfare, Department of Indian System of Medicine and Homeopathy: New Delhi, India, 1999; Volume III, pp. 92-93.

21. Kabra, A.; Sharma, R.; Singla, S.; Kabra, R.; Baghel, U.S. Pharmacognostic characterization of Myrica esculenta leaves. J. Ayurveda Integr. Med. 2017, 10, 18-24. [CrossRef] [PubMed]

22. Dollo, M.; Samal, P.K.; Sundriyal, R.C.; Kumar, K. Environmentally sustainable traditional natural resource management and conservation in Ziro valley, Arunachal Himalaya, India. J. Am. Sci. 2009, 5, 41-52.

23. Kumar, J.K.; Sinha, A.K. Resurgence of natural colourants: A holistic view. Nat. Prod. Res. 2004, 18, 59-84. [CrossRef]

24. Jeeva, S.; Lyndem, F.B.; Sawian, J.T.; Laloo, R.C.; Mishra, B.P. Myrica esculenta Buch.-Ham. ex D. Don.-A potential ethnomedicinal species in a subtropical forest of Meghalaya, northeast India. Asian Pac. J. Trop. Biomed. 2011, 1, S174-S177. [CrossRef]

25. Bhatt, B.P.; Tomar, J.M.S.; Bujarbaruah, K.M. Characteristics of Some Firewood trees and shrubs of the North Eastern Himalayan Region, India. Renew. Energy 2004, 29, 1401-1405. [CrossRef]

26. Kala, C.P. Prioritization of cultivated and wild edibles by local people in the Uttaranchal hills of Indian Himalaya. Indian J. Tradit. Knowl. 2007, 6, 239-243.

27. Gusain, Y.S.; Khanduri, V.P. Myrica esculenta wild edible fruit of Indian Himalaya: Need a sustainable approach for indigenous utilization. Ecol. Environ. Conserv. 2016, 22, S267-S270. 
28. Gupta, R.K. The Living Himalaya; Today and Tomorrow Printers and Publishers: Delhi, India, 1989; Volume 2.

29. Fang, Z.; Zhang, M.; Wang, L. HPLC-DAD-ESIMS analysis of phenolic compounds in bayberries (Myrica rubra Sieb. et Zucc.). Food Chem. 2007, 100, 845-852. [CrossRef]

30. Zhang, W.S.; Li, X.; Zheng, J.T.; Wang, G.Y.; Sun, C.D.; Ferguson, I.B. Bioactive components and antioxidant capacity of Chinese bayberry (Myrica rubra Sieb. and Zucc.) fruit in relation to fruit maturity and post harvesting storage. Eur. Food Res. Technol. 2008, 227, 1091-1097. [CrossRef]

31. Fang, Z.; Zhang, Y.; Lu, Y.; Ma, G.; Chen, J.; Liu, D.; Ye, X. Phenolic compounds and antioxidant capacities of bayberry juices. Food Chem. 2009, 113, 884-888. [CrossRef]

32. Zhou, S.H.; Fang, Z.X.; Lu, Y.; Chen, J.C.; Liu, D.H.; Ye, X.Q. Phenolics and antioxidant properties of bayberry (Myrica rubra Sieb. et Zucc.) pomace. Food Chem. 2009, 112, 394-399. [CrossRef]

33. Lau-Cam, C.A.; Chan, H.H. Flavonoids from Comptonia peregrine. Phytochemistry 1973, 12, 1829. [CrossRef]

34. Jones, J.R.; Lebar, M.D.; Jinwal, U.K.; Abisambra, J.F.; Koren, J.; Blair, L.; O’Leary, J.C.; Davey, Z.; Trotter, J.; Johnson, A.G. The diarylheptanoid (+)-aR,11S-myricanol and two flavones from bayberry (Myrica cerifera) destabilize the microtubule-associated protein tau. J. Nat. Prod. 2011, 74, 38-44. [CrossRef]

35. Semwal, D.K.; Semwal, R.B.; Combrinck, S.; Viljoen, A. Myricetin: A Dietary Molecule with Diverse Biological Activities. Nutrients 2016, 8, 90. [CrossRef]

36. Zhang, X.; Zhang, K.; Wang, Y.; Ma, R. Biological effects study of Myricitrin and relevant molecular mechanisms. Curr. Stem Cell Res. Ther. 2019, 14. [CrossRef]

37. Perkin, A.G.; Hummel, J.J. LXXVI-The colouring principle contained in the bark of Myrica nagi Part I. J. Chem. Soc. Trans. 1896, 69, 1287-1294. [CrossRef]

38. Chauhan, N.S. Medicinal and Aromatic Plants of Himachal Pradesh; Indus Publishing Company: New Delhi, India, 1999; p. 226.

39. Anonymous. Ayurvedic Pharmacopoeia of India, Part 1, Vol III.; Ministry of Health and Family Welfare, Department of Indian System of Medicine and Homeopathy: New Delhi, India, 2007; pp. 90-96.

40. Mozhui, R.; Rongsensashi, L.; Changkija, S. Wild edible fruits used by the tribals of Dimapur district of Nagaland, India, East Himalayan Society for Spermatophyte Taxonomy. Pleione 2011, 5, 56-64.

41. Kirtikar, K.R.; Basu, B.D. Indian Medicinal Plants. Vol. III, 2nd ed.; International book distributors: New Delhi, India, 1999; p. 1699.

42. Osmaston, A.E. A Forest Flora of Kumaon; Bishen Singh Mahinder Pal Singh: Dehradun, India, 1994.

43. Singh, N.P.; Gajurel, P.R.; Rethy, P. Ethnomedicinal value of traditional food plants used by the Zeliang tribe of Nagaland. Indian J. Tradit. Knowl. 2015, 14, 298-305.

44. Gyawali, R.; Hengaju, A.; Thapa, P.M.; Khadka, P.; Sah, R.; Bhandari, S.; Adhikari, S.; Subedi, S.; Shrestha, A.K.; Shrestha, T.M. Antioxidant and Wound Healing Property of Polyherbal Ointment of Nepalese Medicinal Plants. Int. J. Allied Med. Sci. Clin. Res. 2016, 4, 275-283.

45. Shrestha, P.M.; Dhillion, S.S. Medicinal plant diversity and use in the highlands of Dolakha district, Nepal. J. Ethnopharmacol. 2003, 86, 81-96. [CrossRef]

46. Nguyen, X.N.; Phan, V.K.; Chau, V.M.; Bui, H.T.; Nguyen, X.C.; Vu, K.T.; Hoang le, T.A.; Jo, S.H.; Jang, H.D.; Kwon, Y.I.; et al. A new monoterpenoid glycoside from Myrica esculenta and the inhibition of Angiotensin I-Converting Enzyme. Chem. Pharm. Bull. 2010, 58, 1408-1410. [PubMed]

47. Kankanamalage, T.N.M.; Dharmadasa, R.M.; Abeysinghe, D.C.; Wijesekara, R.G.S. A survey on medicinal materials used in traditional systems of medicine in Sri Lanka. J. Ethnopharmacol. 2014, 155, 679-691. [CrossRef] [PubMed]

48. Kuang, K.Z.; Lu, A.M. Myricaceae, Flora Reipublicae Popularis Sinicae; Science Press: Beijing, China, 1979; pp. 1-6.

49. Parmar, C.; Kaushal, M.K. Wild Fruits of the Sub-Himalayan Region. In Myrica nagi; Parmar, C., Kaushal, M.K., Eds.; Kalyani Publishers: New Delhi, India, 1982; pp. 49-53.

50. Dhani, A. Major wild edible fruits used by locals of Garhwal Himalaya. Int. J. Adv. Life Sci. 2013, 6, 145-149.

51. Pundir, S.; Tomar, S.; Upadhyay, N.; Sharma, V. Antioxidant, anti-inflammatory and analgesic activity of bioactive fraction of leaves of Myrica esculenta Buch.-Ham along with its pharmacognostic and chromatographic evaluation. Int. J. Biol. Pharm. Allied Sci. 2015, 4, 6509-6524.

52. Singh, J.; Lan, V.K.; Trivedi, V.P. Pharmacognostic evaluation of Katphala (The bark of Myrica esculenta Buch-Ham). Anc. Sci. Life 1986, 6, 85-87. 
53. Sahu, S.; Sahu, C.R.; Yadav, A.; Rathod, P.; Chaturvedi, S.; Tripathi, R. Review on Myrica esculenta a popular plant of Himalayan region. J. Chem. Pharm. Sci. 2013, 6, 93-97.

54. Anonymous. Data base on Medicinal Plants used in Ayurveda and Siddha, Volume VIII.; CCRAS: New Delhi, India, 2007; p. 207.

55. Mallya, S.V.; Nesari, T.; Kumar, K.N. Pharmacognostic standards of Katphala (Myrica nagi Hook. f. non-Thumb); A potent bark drug used in Indian systems of medicine. J. Sci. Innov. Res. 2016, 5, $135-137$.

56. Panthari, P.; Kharkwal, H.; Kharwal, H.; Joshi, D.D. Myrica nagi: A review on active constituents, biological and therapeutic effects. Int. J. Pharm. Pharm. Sci. 2012, 4, 38-42.

57. Boloor, V.A.; Hosadurga, R.; Rao, A.; Jenifer, H.; Pratap, S. Unconventional Dentistry in India - An Insight into the Traditional Methods. J. Trad. Complement. Med. 2014, 4, 153-158. [CrossRef] [PubMed]

58. Manandhar, N.P. A survey of medicinal plants of Jajarkot district, Nepal. J. Ethnopharmacol. 1995, 48, 1-6. [CrossRef]

59. Nainwal, P.; Kalra, K. Study on the wound activity potential on the aqueous extract of the bark of Myrica esculenta Buch. \&Ham. Int. J. Pharm. Clin. Res. 2009, 1, 85-87.

60. Gaire, B.P.; Subedi, L. Medicinal Plant Diversity and their Pharmacological Aspects of Nepal Himalayas. Pharmacogn. J. 2011, 25, 6-17. [CrossRef]

61. Khan, M.Y.; Sagrawat, H.; Upmanyu, N.; Siddique, S. Anxiolytic Properties of Myrica nagi Bark Extract. Pharm. Biol. 2008, 46, 757-761. [CrossRef]

62. Bich, D.H.; Chung, D.Q.; Chuong, B.X.; Dong, N.T.; Dam, D.T.; Hien, P.V.; Lo, V.N.; Mai, P.D.; Man, P.K.; $\mathrm{Nhu}$, D.T.; et al. The Medicinal Plants and Animals in Vietnam; Hanoi Science and Technology Publishing House: Hanoi, Vietnam, 2004; Volume 1, pp. 612-613.

63. Joshi, A.R.; Edington, J.M. The use of medicinal plants by two village communities in the Central Development Region of Nepal. Econ. Bot. 1990, 44, 71-83. [CrossRef]

64. Sharma, H.K.; Chhangte, L.; Dolui, A.K. Traditional medicinal plants in Mizoram, India. Fitoterapia 2001, 72, 146-161. [CrossRef]

65. Shrestha, N.; Prasai, D.; Shrestha, K.K.; Shrestha, S.; Zhang, X.C. Ethnomedicinal practices in the highlands of central Nepal: A case study of Syaphru and Langtang village in Rasuwa district. J. Ethnopharmacol. 2014, 155, 1204-1221. [CrossRef]

66. Kumari, P.; Joshi, G.C.; Tewari, L.M. Diversity and status of ethnomedicinal trees of Almora district in Uttarakhand, India. Int. J. Biodivers. Conserv. 2011, 3, 298-326.

67. Kichu, M.; Malewska, T.; Akter, K.; Imchen, I.; Harrington, D.; Kohen, J.; Vemulpad, S.R.; Jamie, J.F. An ethnobotanical study of medicinal plants of Chungtia village, Nagaland, India. J. Ethnopharmacol. 2015, 166, 5-17. [CrossRef] [PubMed]

68. Semwal, D.P.; Saradhi, P.P.; Kala, C.P.; Sajwan, B.S. Medicinal plants used by local Vaidyas in Ukhimath block, Uttarakhand. Indian J. Tradit. Knowl. 2010, 9, 480-485.

69. Bhatt, I.D.; Rawat, S.; Badhani, A.; Rawal, R.S. Nutraceutical potential of selected wild edible fruits of the Indian Himalayan region. Food Chem. 2017, 215, 84-91. [CrossRef]

70. Dhyani, P.P.; Dhar, U. Myrica esculenta, Box Myrtle (Kaiphal); Himavikas Occasional Publication; GB Pant Institute of Himalayan Environment and Development: Almora, India, 1994.

71. Pala, N.A.; Negi, A.K.; Todaria, N.P. Traditional uses of medicinal plants of Pauri Garhwal, Uttrakhand. Nat. Sci. 2010, 8, 57-61.

72. Seal, T. Nutritional composition of wild edible fruits in Meghalaya state of India and their ethnobotanical Importance. Res. J. Bot. 2011, 6, 58-67.

73. Chandra, S.; Saklani, S.; Mishra, A.P.; Badoni, P.P. Nutritional evaluation, antimicrobial activity and phytochemical screening of wild edible fruit of Myrica nagi pulp. Int. J. Pharm. Pharm. Sci. 2012, 4, 407-411.

74. Patel, V.G.; Patel, K.G.; Patel, K.V.; Gandhi, T.R. Development of Standardisation parameters and Isolation of Phytomarker Myricetin from stem bark of Myrica esculenta Buch. Ham. Ex d. Don. J. Pharmacogn. Phytochem. 2017, 6, 29-34.

75. Singh, N.; Khatoon, S.; Srivastava, N.; Rawat, A.; Mehrotra, S. Qualitative and quantitative standardization of Myrica esculenta Buch.-Ham. Stem bark by use of HPTLC. J. Planar Chromatogr. 2009, 22, 287-291. [CrossRef]

76. Rawat, S.; Jugran, A.; Giri, L.; Bhatt, I.D.; Rawal, R.S. Assessment of antioxidant properties in fruits of Myrica esculenta: A popular wild edible species in Indian Himalayan Region. Evid. Based Complet. Altern. Med. 2011, 2011, 1-8. [CrossRef] 
77. Wei, Y.; Chang-ming, T.; Xian, L.; Ya, Z.; Li, W.; Liang, L. Study on the chemical constituents of Myrica esculenta. J. Yunnan Univ. (Nat. Sci.) 2011, 33, 453-457.

78. Mann, S.; Satpathy, G.; Gupta, R.K. In-vitro evaluation of bioprotective properties of underutilized Myrica esculenta Buch.-Ham. ex D. Don fruit of Meghalaya. Indian J. Nat. Prod. Resour. 2015, 6, 183-188.

79. Dawang, S.; Zuchun, Z.; Foo, L.Y.; Wong, H. Flavonols from Myrica esculenta bark. Chem. Indus. Forest Prod. 1991, 4, 251-257.

80. Bamola, A.; Semwal, D.K.; Semwal, S.; Rawat, U. Flavonoid glycosides from Myrica esculenta leaves. J. Indian Chem. Soc. 2009, 86, 535-536.

81. Available online: http://www.niscair.res.in/activitiesandservices/products/wealth-ofIndiaFolder2010.pdf (accessed on 15 May 2019).

82. Agnihotri, S.; Wakode, S.; Ali, M. Triterpenoids from the stem bark of Myrica esculenta Buch Ham. World J. Pharm. Pharm. Sci. 2016, 5, 1319-1327.

83. Hui-fen, M.A.; Zheng-liang, Y.; Sang-zi, Z.E.; Yong-jie, L.; De-lu, N.; Zhen, Y.U. GC/MS analysis of volatile components from leaf of Myrica esculenta Buch.-Ham. Guangdong Agric. Sci. 2011, 16, 18.

84. Krishnamoorthy, V.; Seshadri, T.R. A new Proanthocyanidin from the stem bark of Myrica nagi thumb. Tetrahedron 2001, 22, 2367-2371. [CrossRef]

85. Mei, W.D.; Hong, C.J.; Mei, W.Y.; Man, X.; Song, W.Z. Study on ultrasound-assisted extraction of proanthocyanidins from Myrica esculenta Bark. Chem. Ind. Forest Prod. 2009, 29, 105-109.

86. Begley, M.J.; Campbell, R.V.M.; Crombie, L.; Tuck, B.; Whiting, D.A. Constitution and absolute configuration of meta, metabridged, stained biphenyls from Myrica nagi: X-ray analysis of 16-bromomyricanol. J. Chem. Soc. C Org. 1971, 1970, 3634-3642.

87. Malterud, K.E.; Anthonsen, T. 13-oxomyricanol, a new [7.0]-metacyclophane from Myrica nagi. Phytochemistry 1980, 19, 705-707. [CrossRef]

88. Agarwal, K.P.; Roy, A.C.; Dhar, M.L. Triterpenes from the Bark of Myrica esculenta Buch.-Ham. Indian J. Chem. 1963, 1, 28-30.

89. Husain, S.R.; Cillard, J.; Cillard, P. Hydroxyl radical scavenging activity of flavonoids. Phytochemistry 1987, 26, 2489-2491. [CrossRef]

90. Afanaslev, I.B.; Dorozhko, A.I.; Bordskii, A.V. Chelating and free radical scavenging mechanisms of inhibitory action of rutin and quercetin in lipid peroxidation. Biochem. Pharmacol. 1989, 38, 1763-1769. [CrossRef]

91. Torel, J.; Cillard, J.; Cillard, P. Antioxidant activity of flavonoids and reactivity with peroxy radical. Phytochemistry 1986, 25, 383-385. [CrossRef]

92. Pant, G.; Prakash, O.; Chandra, M.; Sethi, S.; Punetha, H.; Dixit, S. Biochemical analysis, pharmacological activity, antifungal activity and mineral analysis in methanol extracts of Myrica esculenta and Syzygiumcumini: The Indian traditional fruits growing in Uttarakhand Himalaya. Indian J. Pharm. Biol. Res. 2014, 2, $26-34$.

93. Middha, S.K.; Kumar, G.A.; Talambedu, U.; Babu, D.; Misra, A.K.; Prakash, L. Evaluation of antioxidative, analgesic and antiinflammatory activities of methanolic extract of Myrica nagi leaves-An animal model approach. Symbiosis 2016, 13, 179-184. [CrossRef]

94. Seal, T. Antioxidant Activity of Some Wild Edible Fruits of Meghalaya State in India. Adv. Biol. Res. 2011, 5, 155-160.

95. Goyal, A.K.; Mishra, T.; Bhattacharya, M.; Kar, P.; Sen, A. Evaluation of phytochemical constituents and antioxidant activity of selected actinorhizal fruits growing in the forests of Northeast India. J. Biosci. 2013, 38, 797-803. [CrossRef]

96. Chen, J.; Wang, Y.; Wu, D.; Wu, Z. Preliminary study on antioxidative and radical scavenging activities of extracts from Myrica esculenta Buch.-Ham. Bark. Chem. Ind. Forest Prod. 2007, S1, 1-7.

97. Rana, R.K.; Patel, R.K. Antioxidant Activity of Bark of Myrica nagi. Int. J. Pharm. Sci. Rev. Res. 2014, 28, 99-101.

98. Patel, K.G.; Bhalodia, P.N.; Patel, A.D.; Patel, K.V.; Gandhi, T.R. Evaluation of bronchodilator and antianphylactic activity of Myrica sapida. Iran. Biomed. J. 2008, 12, 191-196.

99. Patel, K.G.; Rao, N.J.; Gajera, V.G.; Bhatt, P.A.; Patel, K.V.; Gandhi, T.R. Antiallergic activity of stem bark of Myrica esculenta Buch.-Ham. (Myricaceae). J. Young Pharm. 2010, 2, 74-78. [CrossRef]

100. Patel, T.; Ladani, K.; Shah, S. Antiasthmatic activity of aqueous extract of Myrica nagi bark. Int. J. Phytopharm. Res. 2013, 4, 40-45. 
101. Rana, R.K.; Patel, R.K. Pharmacological Evaluation of Antiasthmatic Activity of Myrica nagi Bark Extracts. Antiinflamm. Antiallergy Agents Med. Chem. 2016, 15, 145-152. [CrossRef]

102. Patel, T.; Rajshekar, C.; Parmar, R. Mast cell stabilizing activity of Myrica nagi bark. J. Pharmacogn. Phytother. 2011, 3, 114-117.

103. Saini, R.; Garg, V.; Dangwal, K. Effect of extraction solvents on polyphenolic composition and antioxidant, antiproliferative activities of Himalayan bayberry (Myrica esculenta). Food Sci. Biotechnol. 2013, 22, 887-894. [CrossRef]

104. Syed, S.; Ahmad, M.; Fatima, N.; Mahjabeen; Jahan,, N. Neuropharmacological studies of Myrica nagi bark. Int. J. Biol. Biotechnol. 2013, 10, 553-558.

105. Rawat, S.; Kumar, N.; Kothiyal, P. Evaluate the antidiabetic activity of Myrica esculenta leaves in streptozotocin induced diabetes in rat. Int. J. Univ. Pharm. Biol. Sci. 2013, 2, 510-525.

106. Nayak, B.K.; Deka, P.; Eloziia, N. Assessment of phytochemical \& pharmacological activities of the ethanol leaves extracts of Myrica esculenta Buch. Ham. J. Pharm. Res. 2017, 11, 444-449.

107. Jain, V.K.; Jain, B. Anthihelmintic Activity of ethanolic extract of bark of Myrica esculenta. Int. J. Pharm. Sci. Res. 2010, 1, 129-131.

108. Patel, T.; Dudhpejiya, A.; Sheath, N. Antiinflammatory activity of Myrica nagi Linn. Bark. Anc. Sci. Life 2011, 30, 100-103.

109. Suryawanshi, J.S.; Karande, K.M.; Udugade, B.V. Antibacterial activity of bark and fruits of Myrica nagi. Indian J. Nat. Prod. 2009, 25, 21-23.

110. Shan, B.; Cai, Y.Z.; Brooks, J.D.; Corke, H. The in vitro antibacterial activity of dietary spice and medicinal herb extracts. Int. J. Food Microbiol. 2007, 117, 112-119. [CrossRef]

111. Agnihotri, S.; Wakode, S.; Ali, M. Essential oil of Myrica esculenta Buch. Ham: Composition, antimicrobial and topical antiinflammatory activities. Nat. Prod. Res. 2012, 26, 2266-2269. [CrossRef]

112. Swathi, D.; Prasad, K.V.S.R.G. Antioxidant and antiulcer potential of ethanolic extract of bark of Myrica esculenta in pyloric ligation ulcer model. Int. J. Pharm. Pharm. Sci. 2015, 7, 195-198.

113. Alam, A.; Iabal, M.; Saleem, M.; Ahmed, S.U.; Sultana, S. Myrica nagi attenuates cumene hydroperoxide-induced cutaneous oxidative stress and toxicity in swiss albino mice. Pharmacol. Toxicol. 2000, 86, 209-214. [CrossRef]

114. Samundeeswari, C.; Rajadurai, M.; Periasami, R.; Kanchana, G. Hepatoprotective effect of Herbitars, A polyherbal against $\mathrm{CCl}_{4}$ induced hepatotoxicity in rats. J. Pharm. Res. 2011, 4, 676-679.

115. Patil, S.P.; Pardeshi, M.L.; Ghongane, B.B. Screening for Anti-allergic and Anti-histaminic Activity of Extract of Momordicadioica, Myrica esculenta and Euphorbiahirta in Animal Models. Res. J. Pharmaceu. Biol. Chem. Sci. 2016, 7, 21-28.

116. Sharma, R.; Kabra, A.; Rao, M.M.; Prajapati, P.K. Herbal and Holistic solutions for Neurodegenerative and Depressive disorders Leads from Ayurveda. Curr. Pharm. Des. 2018, 24, 2597-2608. [CrossRef]

117. Rawal, R.S.; Pandey, B.; Dhar, U. Himalayan forest database-Thinking beyond dominants. Curr. Sci. 2003, 84, 990-994.

118. Lohani, N.; Tewari, L.M.; Joshi, G.C.; Kumar, R.; Kishor, K.; Upreti, M.R. Population assessment and threat categorization of endangered medicinal orchid Malaxis acuminata D. Don. From North-West Himalaya. Int. J. Conserv. Sci. 2013, 4, 483-940.

119. Chapin, F.S.; Zavaleta, E.S.; Eviner, V.T.; Naylor, R.L.; Vitousek, P.M.; Reynolds, H.L.; Hooper, D.U.; Lavorel, S.; Sala, O.E.; Hobbie, S.E.; et al. Consequences of changing biodiversity. Nature 2000, 405, 234-242. [CrossRef]

120. Koorbanally, C.; Crouch, N.R.; Mulholland, D.A. The phytochemistry and ethnobotany of the Southern African genus Eucomis (Hyacinthaceae: Hya- cinthoideae). In Phytochemistry: Advances in Research; Imperato, F., Ed.; Research Signpost: Kerala, India, 2006; pp. 69-85.

121. Sharma, R.; Kuca, K.; Nepovimova, E.; Kabra, A.; Rao, M.M.; Prajapati, P.K. Traditional Ayurvedic and herbal remedies for Alzheimers disease from bench to bedside. Expert Rev. Neurother. 2019, 19, 359-374. [CrossRef]

122. Sharma, R.; Martins, N.; Kuca, K.; Chaudhary, A.; Kabra, A.; Rao, M.M.; Prajapati, P.K. Chyawanprash A Traditional Indian Bioactive Health Supplement. Biomolecules 2019, 9, 161. [CrossRef]

(C) 2019 by the authors. Licensee MDPI, Basel, Switzerland. This article is an open access article distributed under the terms and conditions of the Creative Commons Attribution (CC BY) license (http://creativecommons.org/licenses/by/4.0/). 